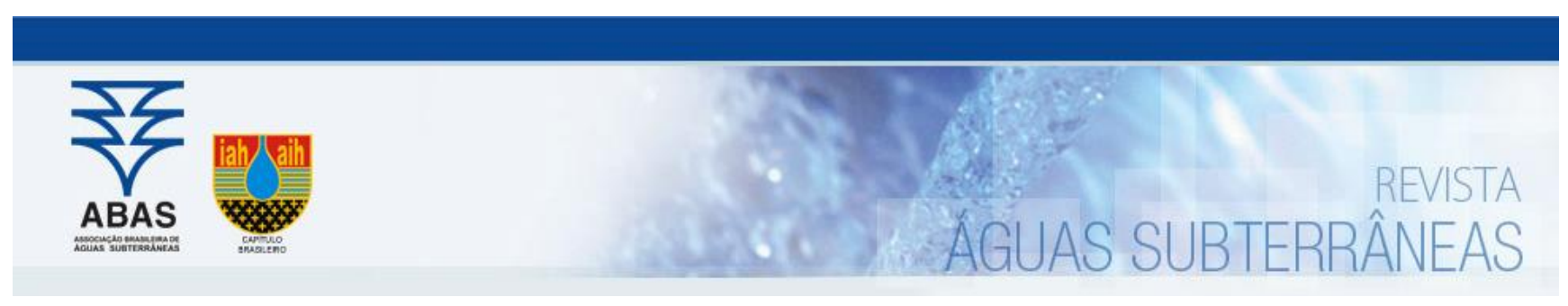

Artigos

\title{
Avaliação da saturação efetiva ar-LNAPL em solos lateriticos com distribuição multimodal de tamanho de poros
}

\section{Evaluation of the effective air-LNAPL saturation in lateritic soils with multimodal pore-size distribution}

\author{
Miguel Alfaro Soto1; Eduardo Patricio dos Santos¹; Chang Hung Kiang1 ${ }^{1}$ \\ 1 Universidade Estadual Paulista (UNESP), Rio Claro, SP \\ \alfaro@rc.unesp.br, eduardod.2007@hotmail.com, chang@rc.unesp.br
}

Resumo

Palavras-chave:

Pressão capilar.

Saturação efetiva.

LNAPL.

Volume específico.

\begin{abstract}
Este trabalho visa avaliar a adequabilidade do modelo desenvolvido pela API (2007) na obtenção indireta de curvas de saturação efetiva para sistemas ar-LNAPL em solos de características lateríticas e com distribuição multimodal de tamanho de poros, tal como os encontrados em diferentes territórios do Brasil. Para esse objetivo, amostras de um solo típico do estado de São Paulo foram submetidas a testes para determinação das saturações efetivas de sistemas ar-água e ar-LNAPL a partir do hanging column test, bem como do papel filtro. Os resultados mostraram curvas de retenção típicas de solo com distribuição de tamanho de poros multimodal, sejam para sistemas ar-água ou arLNAPL. Adicionalmente, a obtenção de curvas de saturação efetiva ar-LNAPL a partir de sistemas ar-água por meio de equações foram coerentes, desde que sejam utilizados modelos de pressão capilar trimodais. Finalmente, tal como apontado por Alfaro Soto e Chang (2015), deve-se ressaltar que a modificação de modelos de previsão de volumes específicos de contaminantes para remediação em solos tropicais é necessária, devido às suas características de retenção diferenciadas.
\end{abstract}

Abstract

Keywords:

Capillary pressure.

Effective saturation.

LNAPL.

Specific volume.

Revisado por pares.

Recebido em: 08/08/2018.

Aprovado em: 25/02/2019.

This paper aims to evaluate the suitability of the model developed by API (2007) in the indirect obtention of effective saturation curves for air-LNAPL systems in soils with lateritic characteristics and multimodal pore-size distribution, such as those found in different Brazilian territories. For this purpose, soil samples typical of the state of São Paulo were tested in order to determine the effective saturations of air-water and air-LNAPL systems from the hanging column test, as well as, filter paper method. The results showed typical retention curves of soils with multimodal pore size distribution, whether for air-water or air-LNAPL systems. Furthermore, obtaining effective air-LNAPL saturation curves from air-water systems were consistent, whenever trimodal capillary pressure models were used. Finally, as pointed out by Alfaro Soto and Chang (2015), it should be emphasized that the modification of prediction models of specific volumes of contaminants for remediation in tropical soils could be necessary due to their differentiated retention characteristics.

DOI: http:/dx.doi.org/10.14295/ras.v33i1.29192

\section{INTRODUÇÃO}

Derramamentos acidentais de derivados do petróleo em superfície é uma das mais comuns formas de contaminação de solos e águas subterrâneas e podem conduzir à formação de Fase Líquida Leve Não Aquosa (LNAPL- Light Non-Aqueous Phase Liquid). Um requisito para o planejamento da remediação de áreas contaminadas com LNAPL é a determinação do volume de LNAPL em subsuperfície.

Primeiras tentativas de estimar o volume de LNAPL a partir de sua espessura medida em poços consideravam a porosidade subsuperficial e a extensão da contaminação por LNAPL (HAMPTON e MILLER, 1988), assumindo uma saturação LNAPL de 1, ou 1 menos a saturação de água residual
(VAN DAM, 1967). No entanto, Schwille (1967), van Dam (1967), entre outros, verificaram que a espessura real do LNAPL na subsuperfície era menor que a espessura do LNAPL em um poço, sendo esta última apenas uma espessura aparente. Estudos (ZILLIOX e MUNTZER, 1975; HALL et al., 1984; BALLESTERO et al., 1994) relacionaram a espessura real de LNAPL no subsolo à espessura aparente de LNAPL, levando em consideração que essa relação dependesse do tamanho do grão da formação subsuperficial, entre outros fatores (NEWELL et al., 1995).

Lenhard e Parker (1990) e Farr et al. (1990) observaram que os modelos acima descritos não eram suficientes e desenvolveram relações fundamentais de saturação e pressão 
capilar para estimar o volume específico de LNAPL a partir da medição de sua espessura em poços. Esses modelos analíticos permitem calcular a variabilidade dos conteúdos de LNAPL e água acima da zona saturada de água descartando a existência das denominadas "panquecas" (modelos antecessores) saturadas de LNAPL.

Atualmente, o modelo desenvolvido por Charbeneau (2003) é muito utilizado e constitui a base conceitual do software do American Petroleum Institute (API, 2007), denominado LNAPL Distribution and Recovery Model (LDRM). O programa permite simular o desempenho de algumas técnicas para recuperação de fase livre decorrente de derramamentos de hidrocarbonetos em águas subterrâneas, permitindo estimativas da distribuição do LNAPL em meios porosos, bem como taxas, volumes e tempos de recuperação de LNAPL.

O modelo API (2007) depende da curva de pressão capilar ou relação saturação/pressão capilar dos sistemas água-LNAPL e ar-LNAPL, e da permeabilidade relativa de LNAPL, entre outros fatores. As funções que descrevem as curvas de pressão capilar são aplicáveis para meios porosos com distribuição de poros unimodal (normal ou log-normal). No entanto, solos podem ter sistemas heterogêneos de poros e consequentemente distribuições de poros multimodais (DURNER, 1994) decorrentes da distribuição granulométrica específica ou à formação de porosidade secundária relacionada a processos genéticos diversos (MALLANTS et al., 1997).

Nesse contexto, este trabalho tem como objetivo avaliar a aplicabilidade da previsão da saturação efetiva de sistemas ar-LNAPL utilizando a formulação matemática contida no software LDRM (API, 2007) quando a porosidade do solo constitui um sistema de poros multimodal, o que pode com duzir a resultados de volumes específicos e de permeabilidade relativa, bem como, estimativas de recuperação de LNAPL diferenciadas.

\section{MÉTODOS TEÓRICOS E EXPERIMENTAIS}

\subsection{Distribuição de LNAPL em poços de monitoramento}

Segundo API (2007), as variáveis envolvidas para as condições de equilíbrio hidrostático entre os fluídos no poço e na formação podem ser resumidas na Figura 1a. As elevações $\mathrm{Z}_{\mathrm{gs}}, \mathrm{Z}_{\mathrm{an}}, \mathrm{Z}_{\mathrm{nw}}, \mathrm{Z}_{\mathrm{aw}}$, correspondem à superfície do terreno, elevação da interface ar-LNAPL no poço, elevação da interface LNAPL-água no poço e elevação do lençol freático sem a presença do LNAPL, respectivamente. Por outro lado, $b_{n}$ representa a espessura do LNAPL no poço de monitoramento e $h_{n}$ e $h_{w}$, as distâncias entre a referência e Zan e Zaw, respectivamente.

Segundo Farr et al. (1990), a identificação dos níveis de elevação no poço de monitoramento associada às curvas de pressão capilar permite o cálculo das distribuição de saturação de LNAPL na formação. A esse respeito, a Figura $1 b$ ilustra a relação entre a saturação do LNAPL com a profundidade, uma vez que guarda relação direta com a pressão capilar.

Na Figura 1b a curva inferior corresponde à curva de pressão capilar LNAPL-água e ilustra a mudança da saturação de água ao longo da espessura do LNAPL no poço de monitoramento. A curva superior corresponde à curva de pressão capilar ar-LNAPL e permite determinar as distribuições de ar, LNAPL e água da zona não saturada.

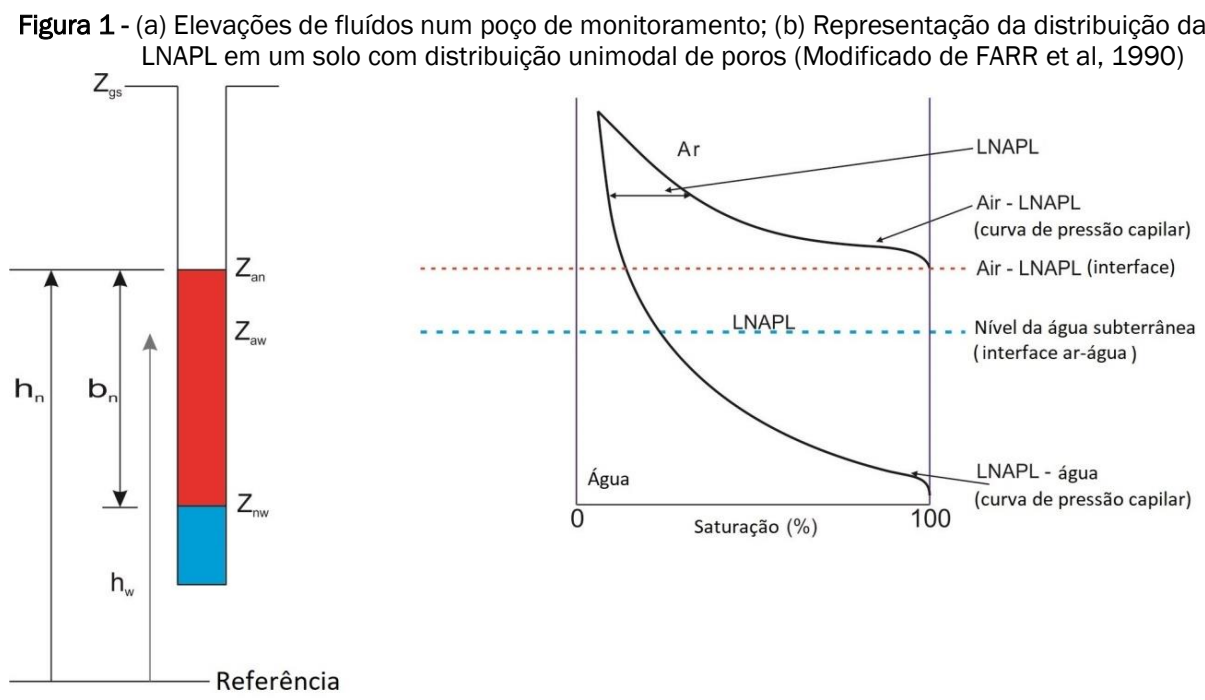

(a)

(b)

A saturação efetiva $\left(\mathrm{S}_{e}\right)$ de um sistema ar-água é comumente representada pela equação de van Genuchten (1980) quando o meio poroso possui uma distribuição de poros unimodal:

$$
S_{e}=\left[1+\left(\alpha h_{c}\right)^{N}\right]^{-M}
$$

Onde $\alpha\left[L^{-1}\right], N$ [-] e M [-] representam os parâmetros de ajuste e $h_{c}$ a pressão capilar.

Um modelo similar à Equação (1) para solos com um sistema de poros multimodal foi formulado por Durner (1994): 


$$
S_{e}=\sum_{i=1}^{k} w_{i}\left[1+\left(\alpha_{i} h\right)^{N_{i}}\right]^{-M_{i}}
$$

Onde $k$ representa o número de sub-sistemas que compõem a total distribuição de poros, wi é o fator de peso de cada sub-curva $\left(0<w_{i}<1\right.$ e $\left.\Sigma w_{i}=1\right)$ que conformam a saturação efetiva com característica multimodal, $\alpha_{i}\left[L^{-1}\right], \mathrm{N}_{\mathrm{i}}[-]$ e $\mathrm{M}_{\mathrm{i}}[-]$ representam os parâmetros de ajuste das sub-curvas no modelo de van Genuchten (1980).

Por outro lado, segundo API (2007) a saturação efetiva total do sistema ar-LNAPL pode ser obtida de forma indireta a partir da Equação (2) da forma seguinte:

$$
\begin{aligned}
& S_{e[t]}(z)=\left(1+\left(\alpha_{[a o]} h_{[a o]}\right)^{N}\right)^{-M} \\
& \alpha_{[a o]}=\frac{\sigma_{a w}}{\sigma_{a o}} \alpha \\
& h_{[a o]}=\left(\rho_{r}\right)\left(z-z_{a o}\right)
\end{aligned}
$$

Onde $S_{\text {e[t] }}$ representa a saturação efetiva total, $\alpha_{[a o]}$ é o fator de escalonamento para o sistema ar-LNAPL; бao e бaw representa a tensão interfacial ar-LNAPL e tensão superficial arágua, respectivamente; $h_{\text {ao }}$ representa a pressão capilar arLNAPL; $\rho_{r}$ representa a densidade relativa e é definido por $\rho_{r}$ $=\rho_{\mathrm{o}} / \rho_{\mathrm{w}}\left(\rho_{\mathrm{o}}\right.$ e $\rho_{\mathrm{w}}$ : densidade do LNAPL e água, respectivamente); $\mathrm{Z}_{\mathrm{ao}}$ corresponde à altura da interface ar-LNAPL (Figura 1a).

No entanto, Alfaro Soto e Chang (2015) observaram que, devido à característica intrínseca de solos lateríticos brasileiros é necessário representar adequadamente a distribuição multimodal de tamanho de poros.

Assim, a saturação efetiva total do sistema ar-LNAPL para solos com distribuição multimodal $\left(\mathrm{S}_{\mathrm{e}[\mathrm{t}] \mathrm{m}}\right)$ de poros pode ser obtida de forma indireta a partir da Equação 2 (Durner, 1994) da forma seguinte:

$S_{e[t] m}(z)=\sum_{i=1}^{k} w_{i}\left(1+\left(\alpha_{[a o]_{i}} h_{[a o]}\right)^{N_{i}}\right)^{-M_{i}}$

$\alpha_{[a o]_{i}}=\frac{\sigma_{a w}}{\sigma_{a o}} \alpha_{i}$

$h_{[a o]}=\left(\rho_{r}\right)\left(z-z_{a o}\right)$

onde $\alpha_{[a n] i}$, representa o fator de "escalonamento" (em semelhança a API, 2007) para o sistema ar-LNAPL de cada subcurva i.

\subsection{Determinação de curvas de retenção ar-água e ar-LNAPL}

O hanging colum test (ASTM, 2003) foi utilizado para determinação da relação grau de saturação versus pressão capilar dos sistemas ar-água e ar-LNAPL, sob o processo de drenagem, isto é, o teste foi iniciado em condições saturadas, com incrementos sucessivos de pressão capilar até um valor máximo de $50 \mathrm{~cm}$ (Figura 2).

Figura 2 - Esquema do Hanging column test empregado
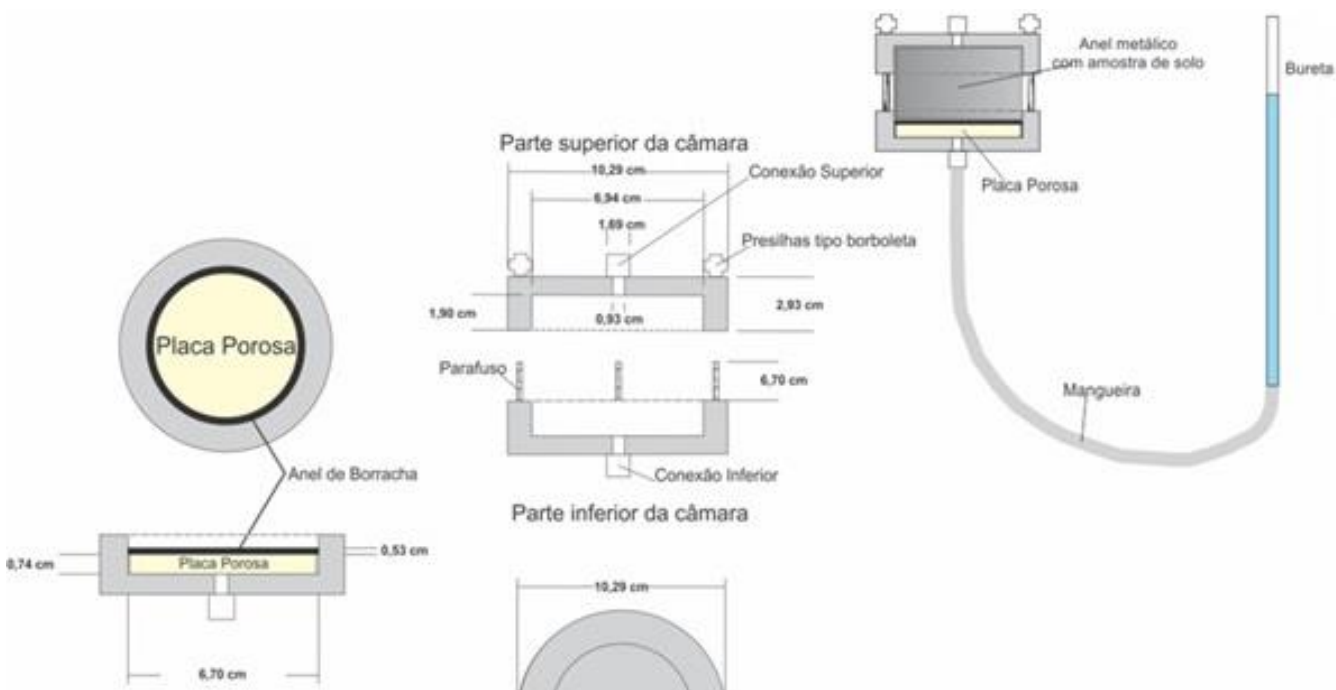

Parte inferior da câmara

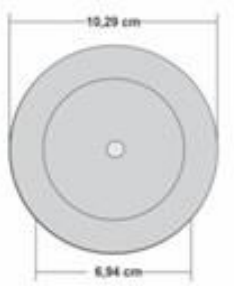


Para realização de testes ar-LNAPL as placas passaram por um tratamento com clorotrimetilsilano, tolueno e metanol para torna-las hidrofóbicas (LENHARD et al., 1988).

O tipo de LNAPL escolhido para os testes foi querosene de aviação. Sua escolha se deve por ser amplamente utilizado e com características básicas similares em diversos países. Tanto para o querosene de aviação como para a água foram determinadas as tensões interfaciais em laboratório pelo método do anel de Du Nouy (marca Kruss, modelo K9-MK1) que consiste em medir a tensão necessária para deslocar um anel de platina da interface ar-água e ar-LNAPL sob condições padronizadas de acordo com normas ASTM (2012) e ASTM (2014).

Adicionalmente, com a finalidade de complementar a curva de pressão capilar foi empregando o método do papel filtro (ASTM, 2003) apenas para o sistema ar-água, visto que este método não é aplicado em sistemas ar-LNAPL. O método consiste em colocar uma amostra de solo em contato com papel filtro de características padronizadas (geralmente Whatman No 42 ou Schleincher e Shuell No 589) dentro de uma atmosfera fechada até que o equilíbrio de potenciais seja alcançado. Após esse período, o teor de umidade do solo é relacionado com sua pressão capilar por meio de equações de calibração baseadas na umidade de equilíbrio do papel filtro.

As amostras de solo foram coletadas em solos residuais da Formação Rio Claro-SP devido a apresentarem distribuição multimodal de tamanho de poros resultantes do fenômeno de estruturação (ALFARO SOTO, 1999, FERNANDES e VILAR, 2011, GRACIOSA et al., 2008, entre outros), e que são de comum ocorrência em solos lateríticos do Brasil.

\section{RESULTADOS E DISCUSSÕES}

\subsection{Curvas de pressão capilar ar-água e ar-LNAPL}

Na execução dos testes, o método de Hanging column test tornou possível medições de curva de saturação efetiva entre 5 e $50 \mathrm{~cm}$, não sendo possível a imposição de maiores valores pressão capilar (recorrente descontinuidade do fluído na mangueira do aparelho), seja no sistema ar-água ou arLNAPL.

Por outro lado, o método do papel filtro permitiu medições de curva de pressão capilar entre 10 e $10^{5} \mathrm{~cm}$, porém a calibração do papel neste método é apenas aplicável a sistemas arágua, não existindo calibração para o sistema ar-LNAPL.

Cabe ressaltar, que em todos os casos os menores valores da curva de pressão capilar foram obtidos por índices físicos, onde a umidade máxima corresponde à umidade de saturação, assumindo uma pressão capilar de 0,1 cm.

A Figura 3 mostra os resultados experimentais das curvas de pressão capilar obtidos para os sistemas ar-água e ar-LNAPL para o solo proveniente da cidade de Rio Claro (São Paulo) que se caracteriza por ser uma areia argilosa.

As curvas de ambos os sistemas mostram semelhança diferindo apenas no grau de saturação entre as faixas que são mensuráveis e comparáveis $(0,1$ a $50 \mathrm{~cm})$, apresentando uma queda súbita de grau de saturação, entre 10 e $20 \mathrm{~cm}$ de pressão capilar. Isto sugere uma distribuição de tamanho de poros diferenciada, provavelmente resultante da estruturação do solo. Por outro lado, a curva do sistema ar-água, sendo mais abrangente, mostra também uma forma peculiar em elevadas pressões capilares (acima de $1 \times 10^{5} \mathrm{~cm}$ ) e que torna difícil o ajuste (Eq. 1) para o modelo de van Genuchten (1980) que é utilizado em solos com distribuição de poros unimodal.

Figura 3 - Dados experimentais: grau de saturação versus pressão capilar para os sistemas ar-água e ar-LNAPL

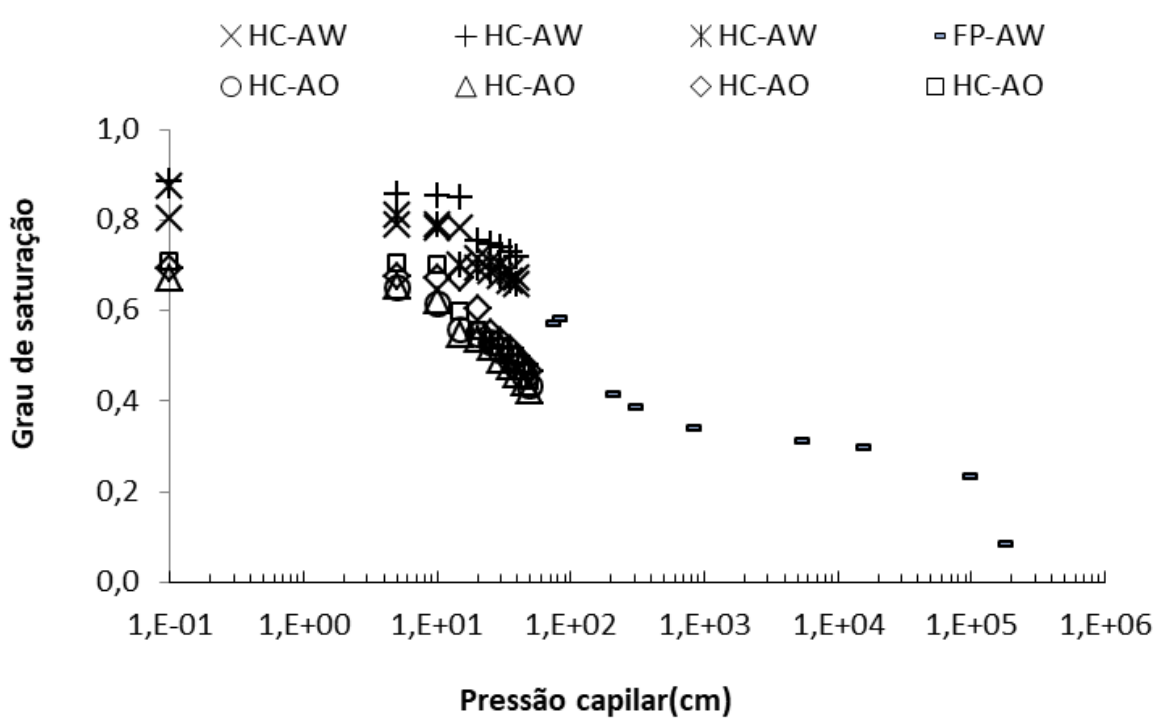

HC:Hanging colum; FP:Papel flitro: AW: Ar-água; AO: ar-LNAPL 
A Figura 4a, mostra os resultados de ajustes uni, bi e trimodal obtidos por regressão não linear (LevenbergMarquardt) utilizando o modelo de van Genuchten (1980) modificado (DURNER, 1994) para uma das amostras de solo com tamanhos de poros com distri- buição multimodal. Na Figura 4 pode-se observar a inadequabilidade dos modelos uni e bimodal para se ajustar aos dados experimentais. Por seu lado, a Figura $4 \mathrm{~b}$, mostra os ajustes uni e multimodais para o sistema Ar-LNAPL na faixa dos dados experimentais.

Figura 4 - Dados experimentais e ajustados da curva de pressão capilar (uma amostra) dos sistemas: A) Ar-água, B) ar-LNAPL

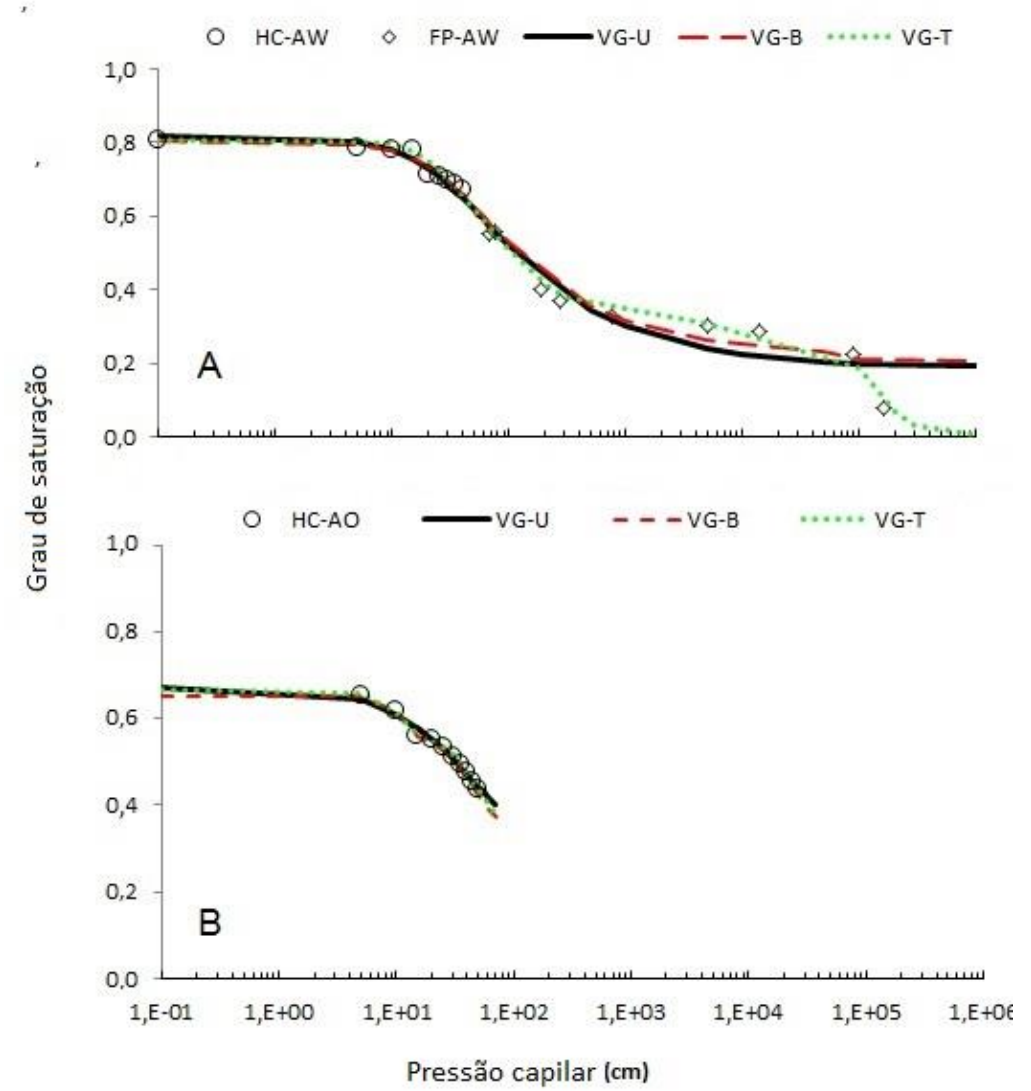

HC:Hanging colum; FP:Papel flitro: AW: Ar-água; AO: Ar-LNAPL; VG-U: van Genuchten unimodal; VG-B: van Genuchten bimodal; VG-T: van Genuchten trimodal.

\subsection{Curvas de saturação efetiva para sistema ar-LNAPL}

As curvas ajustadas pelo modelo unimodal (Eq. 1) de van Genuchten (1980) a partir dos dados experimentais decorrentes de testes em solos não saturados (sistema ar-água) foram transformadas para o sistema ar-LNAPL empregando modelos unimodais (Eq. 3, 4 e 5) da API (2007). Para isto, os valores médios das tensões interfaciais ar-água, ar-LNAPL e densidade relativa, obtidas em laboratório foram: $\sigma_{\mathrm{aw}}=71,6$ dina. $\mathrm{cm}^{-1}, \sigma_{a 0}=27,6$ dina. $\mathrm{cm}^{-1}$ e $\rho_{\mathrm{r}}=0,8$, respectivamente.
Os resultados das curvas ar-LNAPL supracitadas foram comparadas com curvas também ajustadas (Eq. 1) pelo modelo de Van Genuchten (1980), porém para os dados experimentais de testes de curva de pressão capilar medidos em sistema ar-LNAPL, tal como mostrado no item anterior. A comparação teve a finalidade de testar a adequabilidade dos modelos unimodais provenientes da API (2007), quando utilizados em solos com características multimodais. Os resultados são mostrados na Figura 5. 
Figura 5 - Saturação efetiva do sistema Ar-LNAPL versus Pressão capilar obtidos direta (Eq.1) e indiretamente (Eq. 2, 3 e 4 )

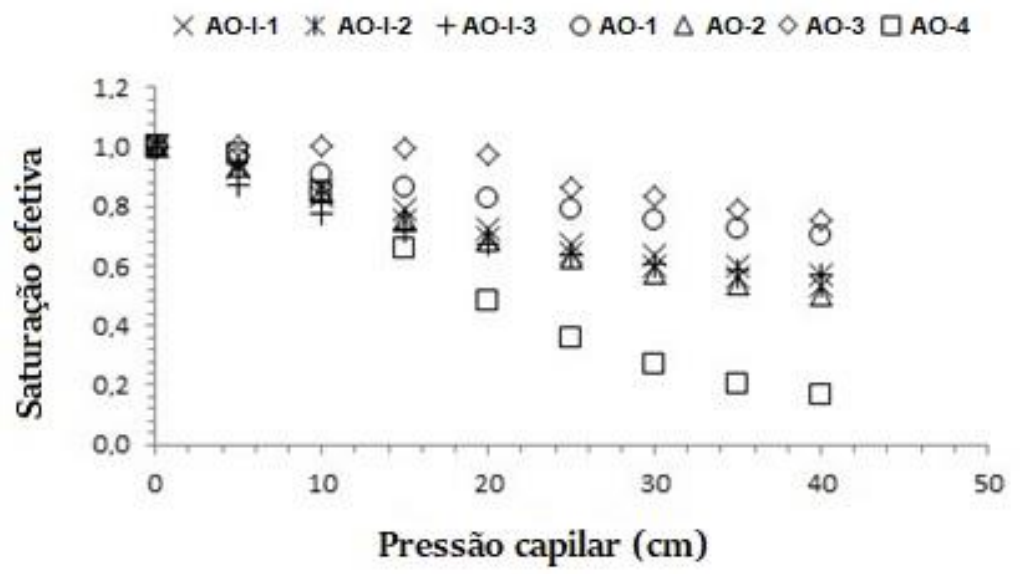

AO: Ar-LNAPL; I: obtido indiretamente com equações (3 à 5)

A Figura 5 mostra que a previsão pelo método unimodal da API (2007) poderia ser aceitável, visto que permite resultados dentro da faixa de dispersão da saturação efetiva obtidos por ajuste (van Genuchten, 1980) a partir de dados experimentais do sistema ar-LNAPL. No entanto, tal como observado na Figura 4, o modelo unimodal não permite ajustes adequados em sistema ar-água em toda a faixa da pressão capilar, o que sugere que, a previsão do sistema arLNAPL não seria representativa.
Diante desta incerteza, foi ampliada a mesma análise comparativa, porém ajustando dados experimentais de sistemas ar-LNAPL por modelos para distribuição de poros bi e trimodais (Eq. 2) e comparando com resultados de previsão de curvas de saturação ar-LNAPL com o método da API (2007) adaptado para solos com distribuição de poros multimodais, segundo equações (Eq.6, 7 e 8) sugeridas por Alfaro Soto e Chang (2015). Os resultados médios (incluindo do modelo unimodal), são confrontados e mostrados na Figura 6.

Figura 6 - Saturação efetiva do sistema Ar-LNAPL (AO-I) obtida indiretamente (Eq.3,4 e 5 para modelo unimodal e Eq.6, 7 e 8 para modelo bi e trimodal) versus Saturação efetiva do sistema Ar-LNAPL (AO) obtida diretamente (Eq.1 para o modelo unimodal e Eq.2, para modelos bi e trimodal).

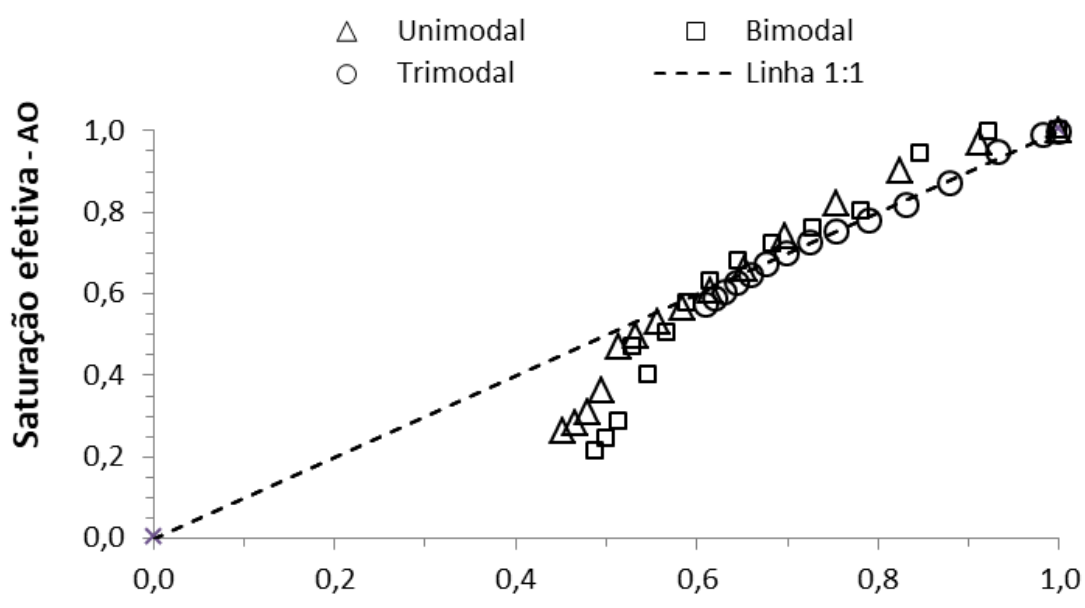

Saturação efetiva - AO-I

Os valores médios das saturações efetivas evidenciam que os modelos indiretos uni (Eq.3,4 e 5) e até bimodais (Eq.6, 7 e 8) podem subestimar em até 1,1 vezes a estimativa de saturação efetiva em baixas pressões capilares (saturações efetivas entre 1 e 0,7) e em contrapartida, superestimá-los em até 2,2 vezes em altas pressões capilares (saturações abaixo de 0,5 aproximadamente) na faixa de pressão capilar avaliada. Por outro lado, a previsão da saturação efetiva do sistema ar-LNAPL empregando o modelo trimodal foi a mais adequada, reproduzindo de melhor forma os dados experimentais.

\section{CONCLUSÕES}

A saturação efetiva do sistema ar-LNAPL obtida indiretamente nos modelos da API (2007) podem não ser adequados quando utilizados em solos cuja distribuição de tamanho de poros seja multimodal. 0 presente trabalho mostrou que 
foram necessários modelos trimodais para minimizar erros de estimativas que podem sobrestimar ou subestimar os graus de saturação efetivas dependendo da magnitude da pressão capilar.

A importância da determinação adequada do grau de saturação efetiva do LNAPL está relacionada com o grau a saturação efetiva total $\left(\mathrm{S}_{\mathrm{e}[\mathrm{t}]}\right)$ e consequentemente, com a determinação dos volumes específicos recuperáveis de LNAPL, sendo este último, indispensável nos projetos de remediação de águas subterrâneas contaminadas por hidrocarbonetos em subsuperfície. Assim, estes resultados servem de alerta para estudos adicionais com a finalidade de estabelecer se as diferenças aqui encontradas são capazes de ocasionar variações significativas de tal forma que, solos de características lateríticas, como os encontrados no Brasil, demandem estimativas específicas para nosso meio, tal como a apresentada neste trabalho.

\section{REFERÊNCIAS}

ALFARO SOTO, M. A.; CHANG H. K. Behaviour of LNAPL in soils with multimodal pore-size distribution. In: PANAMERICAN CONFERENCE ON SOIL MECHANICS AND GEOTECHNICAL ENGINEERING, 15, 2015. Proceedings... Buenos Aires, Argentina, p. 2284-2291, 2015.

ALFARO SOTO, M. A. Estudo da condutividade hidráulica em solos não saturados. 1999, 120p. Dissertação (Mestrado em Geotecnia) - Departamento de Geotecnia, Universidade de São Paulo, Escola de Engenharia de São Carlos, São Carlos, 1999.

AMERICAN SOCIETY FOR TESTING MATERIALS. ASTM $-D$ 6836-16. Standard test method for determination of the soil water characteristic curve for desorption using hanging column, pressure extractor, chilled mirror hygrometer or centrifugue, 2016.

AMERICAN SOCIETY FOR TESTING MATERIALS. ASTM $-D$ 1331-14. Standard test method for surface and interfacial tension of solutions of surface-relative agents, and related materials, 2014.

AMERICAN SOCIETY FOR TESTING MATERIALS. ASTM $-D$ 971-12. Standard method for interfacial tension of oil against water by the ring method, 2012.

AMERICAN SOCIETY FOR TESTING MATERIALS. ASTM $-D$ 5298-03. Standard test method for measurement of soil potential (suction) using filter paper. West Conshohocken, PA, 2003.

AMERICAN PETROLEUM INSTITUTE - API. Light Non-Aqueous Phase Liquid Distribution and Recovery Model (LDRM), API Publication 4760, 2007.

BALLESTERO, T. P.; FIEDLER, F. R.; KINNER, N. E. An investigation of the relationship between actual and apparent gasoline thickness in a uniform sand aquifer. Ground Water, 32, pp. 708-718, 1994.

CHARBENEAU, R, J. Models for design of free-product recovery systems for petroleum hydrocarbon liquids. API Publication Number 4729, American Petroleum Institute, Washington D.C, 2003.
DURNER, W. Hydraulic conductivity estimation for soils with heterogeneous pore structure, Water Resources Research, v. 30, p. 211-223, 1994

FARR, A. M., HOUGHTALEN, R. J., AND MCWHORTER, D. B. Volume estimation of light nonaqueous phase liquids in porous media, Ground Water, v. 28, n. 1, p. 48-56, 1990.

FERNANDES, M. A.; VILAR, O. M. Medida da condutividade hidráulica em solo não saturado por meio do infiltrômetro de disco. In: SIMPÓSIO BRASILEIRO DE SOLOS NÃO SATURADOS, 7., 2011. Anais... Pirenópolis, v. 1, p. 47-55.

GRACIOSA, M. C. P.; MENDIONDO, E. M.; CHAUNDHRY, F. H. Simulação hidráulica de trincheiras de águas pluviais. Revista Brasileira de Recursos Hídricos, v.12, n. 2, p.89-99, 2008.

HALL, R. A.; BLAKE, S. B.; CHAMPLIN, JR. S. C. Determination of hydrocarbon thickness in sediments using borehole data. In: NATIONAL SYMPOSIUM ON AQUIFER RESTORATION AND GROUND WATER MONITORING, 4.,1984. Proceedings... National Water Well Assoc, 1984, p. 300-304.

HAMPTON, D. R.; MILLER, P. D. G. Laboratory investigation of the relationship between actual and apparent product thickness in sands. In: CONF. PETROLEUM HYDROCARBONS AND ORGANIC CHEMICALS IN GROUND WATER: PREVENTION, DETECTION AND RESTORATION, National Water Well Assoc, 1988 , p. $157-181$.

LENHARD, R. J.; DANE, J.; PARKER, J. C.; KALUARCHCHI, J. J. Measurement and simulation of one-dimensional transient three-Phase flow for monotonic liquid drainage. Water Resources Research, v. 24, 6, p.853-863, 1988.

LENHARD, R. J., AND PARKER, J. C. Estimation of free hydrocarbon volume from fluid levels in monitoring wells, Ground Water, v. 28, n. 1, p.57-67, 1990.

MALLANTS, D.; TSENG, P. H.; TORIDE, N.; TIMMERMAN, A.; FEYEN, J. Evaluation of multimodal hydraulic functions in characterizing a heterogeneous field soil. Journal of hydrology, v. 95, p.172-199, 1997.

NEWELL, C. J.; ACREE, S. D.; ROSS, R. R.; HULING, S. G. Light nonaqueous phase liquids. U.S.EPA, R.S. Kerr Environ. Res. Lab. Ada OK. EPA/540/S-95/500, p. 28, 1995.

SCHWILLE, F. Petroleum contamination of the subsoil: a hydrological problem. Brighton (Ed.), The Joint Problems of the Oil and Water Industries: In: SYMPOSIUM HELD AT THE HOTEL METROPOLE, 1987. Proceedings... Brighton, 18-20 January 1967, Institute of Petroleum, London, 1967, p. 2354.

VAN DAM, J. The migration of hydrocarbons in a waterbearing stratum. The Joint Problems of the Oil and Water Industries: In: SYMPOSIUM HELD AT THE HOTEL METROPOLE, Proceedings... Brighton, 18-20, Institute of Petroleum, London, 1967, p. 55-96.

VAN GENUCHTEN, M. TH. A closed-form equation for predicting the hydraulic conductivity of unsaturated soils. Soil. Sci. Soc. Am. J, v. 44, p.892-898, 1980.

ZILLIOX, L.; MUNTZER, P. Effects of hydrodynamic processes on the development of ground-water pollution: Study on physical models in a saturated porous medium. Progress in Water Tech., v. 7, p. 561-568, 1975. 